\title{
The search for dark matter via strong lens inversions of galaxy clusters using genetic algorithms
}

\section{J. Liesenborgs*}

EDM, Universiteit Hasselt, Wetenschapspark 2, B-3590, Diepenbeek, Belgium

E-mail: jori.liesenborgseuhasselt.be

\section{S. De Rijcke; H. Dejonghe}

Sterrenkundig Observatorium, Universiteit Gent, Krijgslaan 281, S9, B-9000, Gent, Belgium

\section{P. Bekaert}

EDM, Universiteit Hasselt, Wetenschapspark 2, B-3590, Diepenbeek, Belgium

\begin{abstract}
Gravitational lensing provides a direct means for measuring the masses of galaxies and galaxy clusters. Provided that enough constraints are available, one might even hope to obtain a handle on the precise distribution of the mass, which in turn may reveal information about the spatial distribution of the dark matter. We present an approach using genetic algorithms, allowing the user to 'breed' solutions which are compatible with available strong lensing data. The procedure allows various types of constraints to be used, including positional information, null-space information, and time-delay measurements. The method is non-parametric in the sense that it does not assume a particular shape of the mass distribution. This is accomplished by placing circularly symmetric basis functions - projected Plummer spheres - on a dynamic grid in the lens plane. Using simulations, we show that our procedure is able construct a mass distribution and source positions that are compatible with a given set of observations. We discuss the degeneracies that are inherent to lens inversion (and hence any lens inversion technique) and that limit the potential of strong lensing to yield precise estimates of the dark-matter distribution. We show how these degeneracies cause most of the differences between inversions of the same lensing cluster by different authors, using the famous cluster $\mathrm{Cl} 0024+1654$ as a working example.
\end{abstract}

Identification of dark matter 2008

August 18-22, 2008

Stockholm, Sweden

\footnotetext{
*Speaker.

${ }^{\dagger}$ Postdoctoral Fellow of the Fund for Scientific Research - Flanders (Belgium)(F.W.O)
} 


\section{Introduction}

The gravitational lens effect depends on the total mass distribution - both luminous and dark - of the deflecting object, thereby providing a direct means for determining its mass. Here, we focus on the strong lens effect, in which the deflection is powerful enough to cause a single source to be transformed into several images of the same object. Clearly, the precise configuration of the images will depend on the precise mass distribution of the lens. Therefore one might hope to obtain a handle on said mass distribution by looking for solutions which are compatible with the observed images, i.e. by trying to invert the lens. In this article, we present an overview of our work on gravitational lens inversion.

\section{Lensing formalism}

The gravitational lens effect is described by the lens equation (2.1), which relates the image plane ( $\theta$-space) to the source plane ( $\beta$-space)

$$
\vec{\beta}(\vec{\theta})=\vec{\theta}-\frac{D_{\mathrm{ds}}}{D_{\mathrm{s}}} \overrightarrow{\hat{\alpha}}(\vec{\theta})
$$

The image plane describes what one will see because of the lens effect; the source plane describes what one would see without the deflection of light rays. The deflection itself is described by the bending angle $\overrightarrow{\hat{\alpha}}$, which depends on the projected mass distribution $\Sigma$ of the lens. The distances $D_{\mathrm{ds}}$ and $D_{\mathrm{s}}$ describe the angular diameter distances between lens and source, and observer and source respectively. A thorough review of the lensing formalism can be found in [1].

\section{Inversion method}

Ideally, one would like to avoid making assumptions about the shape of the lensing mass distribution, i.e. one would like to have a non-parametric inversion method. In practice, this is approximated by building the shape of the mass distribution from a large number of basis functions. Inverting the lens then corresponds to finding appropriate weights for this set of basis functions. In our method, the user has to specify a square-shaped area in which the procedure will try to reconstruct the mass density. Initially, this area is subdivided into smaller grid cells in a uniform way and to each grid cell, a projected Plummer sphere [2] is associated. A genetic algorithm is then used to optimize the weights of these basis functions. Based on this initial solution, a new grid is constructed, in which regions containing a large fraction of the total mass are subdivided further. This new grid is then used to find a new estimate of the mass distribution and this procedure can be repeated until an acceptable reconstruction is found. A similar dynamic grid system is used in [3].

The optimization of the weights of the Plummer basis functions is done using a genetic algorithm, an optimization strategy inspired by the theory of evolution by Darwin. In essence, one tries to 'breed' solutions to a problem. To do so, one starts with an initial 'population', a set of randomly initialized trial solutions. Using this first population, a new one is created by combining and cloning trial solutions of the existing population, and by introducing some mutations. This simple routine is then repeated over and over until an acceptable solution is reached. A vital part of 
the method is the application of selection pressure: one would like to make sure that trial solutions which are deemed better than others, have a better chance in creating more offspring. This will make sure that the genetic algorithm evolves towards increasingly better solutions.

To be able to apply selection pressure, one needs a way to determine how well suited a trial solution is: one needs a fitness measure. In our method, the images of each source are projected back onto their source planes, using the lens equation (2.1) which corresponds to the trial mass map. If the correct lens were to be used, the back-projected images of a source would overlap perfectly. An incorrect lens on the other hand, will project the images onto slightly different regions in the source plane. For this reason, we use the amount of overlap between the backprojected images of a source as the fitness measure.

This approach works well if many sources have multiple images, as is shown in figure 1 using simulated data (see [ $₫$ for additional information). However in the case of only one or a few sources, the solu-
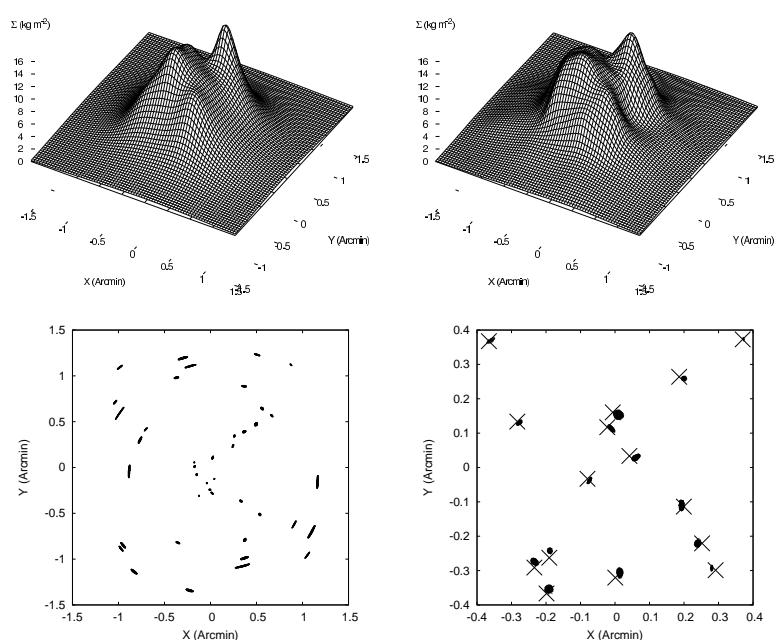

Figure 1: Left panel: an artificial mass map (top) is used to generate simulated images (bottom). Right panel: the images from the left panel are used as input into our inversion procedure, which reconstructs the mass map in this panel (top). When the input images are projected back onto the source plane, the source shapes shown in the lower part are reconstructed. The actual source positions are marked by crosses.

tions which are generated are often not compatible with the observed situation. The reason is clear: while we made sure that the images are projected onto the same region, nothing was done to avoid projecting other parts of the image plane into that very region. If this happens, additional (unobserved) images will be predicted. To avoid this, we need an extra fitness criterion, which takes the information from the null space, i.e. the region in which no images are observed, into account. Fortunately, genetic algorithms are able to optimize several fitness measures simultaneously; they are then referred to as multi-objective genetic algorithms. In a similar way, it is possible to take additional constraints into account (e.g. time delay information). For more detailed information about how the null space is used, we refer to [⿰]𠃌 ; a detailed account of multi-objective genetic algorithms can be found in [6].

When this inversion method is applied to the famous cluster lens $\mathrm{Cl} 0024+1654$, the result in figure 2 (left and middle panel) is obtained. The images of two sources were used; the positions of these images are marked with the letters A and B respectively. The reconstruction shows a good general agreement with the light originating from the cluster: the largest fraction of the cluster mass coincides with the location of the central cluster galaxies. The two galaxies in the lower left corner of the left hand figure are also retrieved automatically. Aside from this general agreement, there is also the interesting feature marked by the letter P. At that location, the algorithm seems to suggest the presence of a peak in the mass density, in a region where only little cluster light can be seen. For more information about the inversion of this cluster, the reader is referred to [7]. 

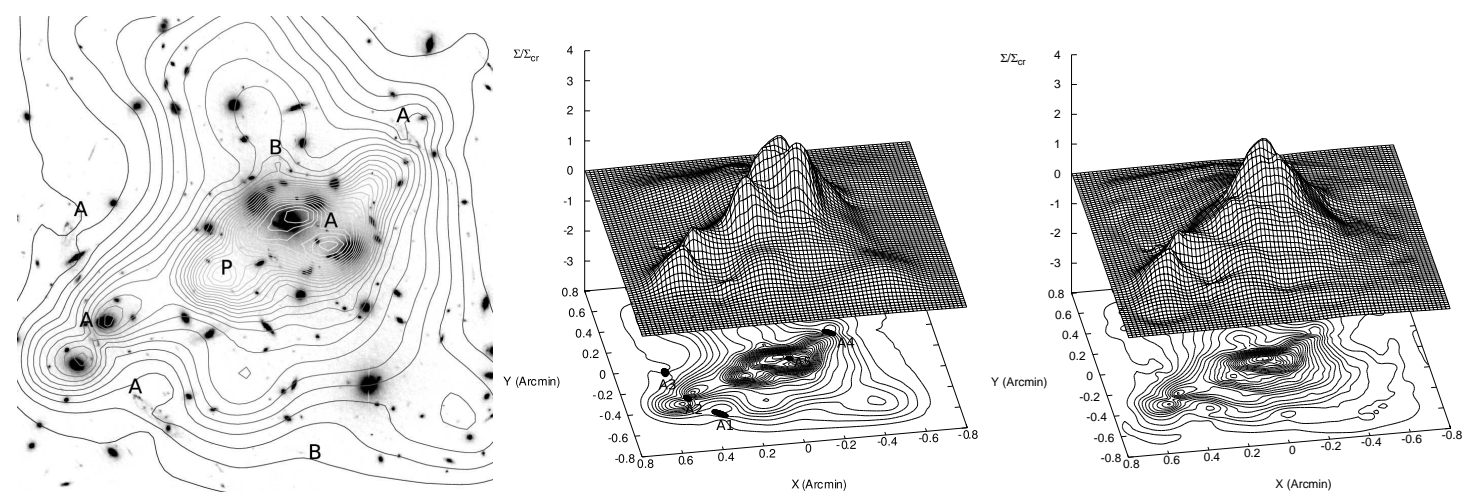

Figure 2: Left panel: reconstructed mass density as a contour map on top of the ACS image. Locations marked by A and B correspond to the input images of two sources. At the location marked by $\mathrm{P}$, there is a clear peak in the mass map where little cluster light can be seen. Center panel: 3D plot of the reconstructed mass distribution, which also clearly shows the peak around P. Right panel: after redistributing the mass (see text) this mass map is obtained, which is equally compatible with the observed situation.

\section{Degeneracies}

To know which features of a reconstructed mass map can be considered as true features, it is important to understand the degeneracies present in lens inversions. Suppose one has a mass reconstruction which is compatible with the observed images of a single source. Then, it is possible to create an infinite amount of other mass distributions which are equally acceptable. One only needs to rescale the original mass map and add a mass sheet of a

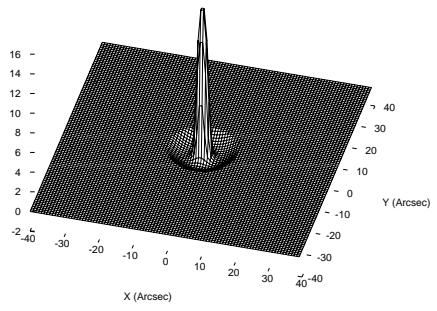

Figure 3: Example of a circularly symmetric mass distribution with a total mass of zero beyond a specific radius. specific density (e.g. [8]). The overall effect of this operation is that the same image plane will then be projected onto a scaled version of the source plane by the new mass map. It is shown in [9] that this mass-sheet or steepness degeneracy can be generalized to the case when one has two or more sources at different redshifts. However, instead of using a mass sheet in the construction of a degenerate solution, a more complex distribution is required.

For a circularly symmetric mass distribution, the bending angle $\overrightarrow{\hat{\alpha}}$ at a specific radius only depends on the total enclosed mass within that radius. This implies that if the total mass stays zero beyond a certain radius, no gravitational lens effect will be generated in that region. Figure 3 shows a mass distribution with this property. As an example, consider the mass distribution in the upper left panel of figure 4 , which transforms a circular source into five distinct images, as is shown in the lower left panel. Inside the circle on the same figure, we then add the 'monopole' from figure 3. Because the non-zero part of the monopole does not overlap with any of the images, the lens equation at the location of the images will not be affected. Indeed, when we use the resulting mass map (upper right panel of figure (4) to recalculate the images of the circular source, the exact same images are found. This example shows explicitly how, using this monopole degeneracy, a considerable mass peak can be easily introduced into an existing mass map which is compatible with the observed images. For the case of $\mathrm{Cl} 0024+1654$, a large number of monopole basis functions were used, making sure that none of them overlapped with the images. The weights of the 
basis functions were then optimized to minimize the overall gradient of the mass map. The result of this procedure can be seen in the right panel of figure 20; note that this procedure automatically erased the mysterious mass peak.

\section{Conclusion}

In this article we have described the basic ideas underlying our lens inversion procedure and we illustrated the method using the cluster lens $\mathrm{Cl} 0024+1654$. It is clearly important to understand degeneracies, otherwise, for example, one might have easily interpreted the mass peak found in the reconstruction of $\mathrm{Cl} 0024+1654$ as dark matter. The monopole and mass-sheet degeneracy together can easily create a wide variety of mass maps which are all compatible with the observed situation. The mass-sheet degeneracy becomes increasingly hard to break as sources are added; the monopole degeneracy on the other hand always allows mass in between the images to be redistributed. It is then clear that for accurate non-parametric mass maps, many multiply imaged sources are necessary, since it is the density of the images which determines the resolution that can be attained.

\section{References}

[1] P. Schneider, J. Ehlers, E. E. Falco, Gravitational Lenses, Springer-Verlag, Berlin, 1992

[2] H. C. Plummer, On the problem of distribution in globular star clusters, MNRAS 71 (1911) 460

[3] J. M. Diego, P. Protopapas, H. B. Sandvik, M. Tegmark, Non-parametric inversion of strong lensing systems, MNRAS 360 (2005) 477

[4] J. Liesenborgs, S. De Rijcke, H. Dejonghe, A genetic algorithm for the non-parametric inversion of strong lensing systems, MNRAS 367 (2006) 1209

[5] J. Liesenborgs,S. De Rijcke, H. Dejonghe, P. Bekaert, Non-parametric inversion of gravitational lensing systems with few images using a multi-objective genetic algorithm, MNRAS 380 (2007) 1729

[6] K. Deb, Multi-Objective Optimization Using Evolutionary Algorithms, John Wiley \& Sons Inc., New York, 2001

[7] J. Liesenborgs, S. De Rijcke, H. Dejonghe, P. Bekaert, Non-parametric strong lens inversion of Cl 0024+1654: illustrating the monopole degeneracy, MNRAS 389 (2008) 415

[8] P. Saha, Lensing Degeneracies Revisited, AJ 120 (2000) 1654

[9] J. Liesenborgs, S. De Rijcke, H. Dejonghe, P. Bekaert, A generalisation of the mass-sheet degeneracy producing ring-like artefacts in the lens mass distribution, MNRAS 386 (2008) 307 